\title{
MORFOLOGIA DOS ÓRGÃOS GENITAIS FEMININOS DO MUÇUÃ (Kinosternon scorpioides)
}

\author{
(Morphology of the female genital organs from muçua (Kinosternon scorpioides)
}

\author{
MACHADO JÚNIOR, A.A.N.. ${ }^{1}$ SOUSA, A.L.'; SANTOS, F.C.F. ${ }^{3}$; PEREIRA, J.G. ${ }^{4}$
}

'Doutorando em Ciência Animal, Universidade Federal do Piauí; ²Departamento das Clínicas Veterinárias, Universidade Estadual do Maranhão;

${ }^{3}$ Mestranda em Ciência Animal, Universidade Federal do Piauí; ${ }^{4}$ Departamento de Patologia, Universidade Estadual do Maranhão.

\begin{abstract}
RESUMO - Estudaram-se os órgãos genitais femininos de dez exemplares de muçuã (Kinosternon scorpioides) adultos, visando conhecer a anatomia, topografia e possíveis variações anatômicas. Os animais foram submetidos à eutanásia sacrificados com dose letal (3ml) de tiopental sódico 2,5\%, seguido de abertura da cavidade celomática e dissecação macroscópica, auxiliada por lupa. Os resultados mostraram os órgãos genitais femininos do muçuã formados por um par de ovários e ovidutos. Os ovários apresentavam-se constituídos por folículos em diferentes estágios de desenvolvimento, sustentados pelo mesovário, enquanto que os ovidutos estavam fixados pelo mesoviduto e divididos em cinco segmentos, infundíbulo, magnum, istmo, útero e vagina, esta última inserida na cloaca, compartimento comum aos aparelhos urogenital e digestório. Com base nos achados, conclui-se que os órgãos genitais femininos do muçuã são funcionais bilateralmente, porém, semelhança anatômica ao oviduto esquerdo das aves.
\end{abstract}

Palavras-chave: Anatomia; Genital feminino; Muçuã.

\begin{abstract}
ABSTACT - With the aim to study and describe the anatomy, the topography and possible anatomic variations of the female genital organs of the mud turtle Kinosternon scorpiedes, ten adult animals were used for such purpose. The animals were sacrificed by an overdose $(3 \mathrm{ml})$ of sodium pentobarbital $2,5 \%$. After the sacrifice, the coelom cavity has been opened and with the aid of a magnifying glass the parts were dissected and photographed. The results did shown that the genital organs of Kinosternon scorpiedes female are formed by a pair of ovaries and one pair of oviducts. The ovaries showed up as being constitued by follicles in different stages of development sustained by a mesovary ligament. The oviducts were divided into five segments, infundibulum, magnum, isthmus, uterus and vagina, fixed by a mesoviduct that begins next to the ovaries and goes to insent itself into the cloaca, this last one being common to the urogenital and digestive systems. These findings allowed the conclusion that this species of mud turtle female genital organs display a bilateral way of function, similar to that of the birds left oviduct.
\end{abstract}

Key-words: Anatomy; Female genital; Muçua.

\section{Introdução}

O muçuã (Kinosternon scorpioides) é uma das tartarugas menos estudadas pela ciência e uma das mais ameaçadas de extinção (FIGURA 1A). É um pequeno réptil nativo da Região Amazônica, pertencente à Ordem Testudines, Família Kinosternidae, distribuído desde a Costa Rica até o norte da Argentina e Brasil. No Maranhão, é encontrado principalmente na região da Baixada Maranhense, onde é chamado de Jurará (PEREIRA, 2000).

Vários fatores põem em risco a sobrevivência dessa espécie, sendo um dos principais o fato de animais serem consumidos como iguaria na culinária maranhense e paraense. Associado a isso, queimadas, desmatamentos e caça indiscriminada contribuem para a diminuição da população de muçuãs na natureza, sendo estes os principais motivos pelos quais sua captura é proibida pelo Instituto Brasileiro do Meio Ambiente e dos Recursos Renováveis (IBAMA).

As espécies viventes de répteis, estimadas em 6000, descendem de um grande grupo de vertebrados, que predominaram durante a Era Mesozóica. O sucesso que eles tiveram naquela época, geralmente, tem sido atribuído ao desenvolvimento de um novo método de proteção embrionária, a casca envolvendo o vitelo, sendo esta suficientemente porosa para permitir a passagem de gases respiratórios, e sólida o bastante para proteger o embrião contra as adversidades ambientais (ORR, 1986).

Os répteis possuem dimorfismo sexual externo e uma fecundação interna. Reproduzem-se através de ovos, que em alguns ofídios se abrem ainda no corpo da mãe. Segundo HILDEBRAND (1995), nos répteis e aves o trato genital das fêmeas varia muito nos detalhes estruturais, porém uma vez que o aparelho reprodutor das duas classes possuem ovos grandes, são semelhantes na forma geral.

A escassa literatura sobre a morfologia dos órgãos genitais femininos do Kinosternon scorpioides motivou a buscar o embasamento teórico na classe dos répteis e, em especial, a ordem dos quelônios, representados pelas tartarugas. Para tanto, NOBLE e NOBLE (1940) e ASHLEY (1969) informam que o aparelho genital feminino das tartarugas é composto por um par de 
ovários e um de ovidutos, sendo assim também descrito por BAROUDI (1970), SANDLEIR (1973) e STORER et al. (2000) para os répteis.

Os ovários nos répteis se apresentam como sacos cheios de ovos, localizados caudalmente na cavidade pleuroperitoneal; essas informações foram prestadas por ASHLEY (1969) e ANDREW e HICKMAN (1974), descrição também idêntica de ELLENPORT (1986) e DYCE et al. (1997) para as aves. Quanto à fixação dessas gônadas, ASHLEY (1969) e BLACKBURN (1998) informam que elas estão suspensas ao teto da cavidade pelo mesovário.

Sobre a sua posição, os ovários das tartarugas, estão dispostos simetricamente no interior da cavidade pleuroperitoneal (OWEN, 1866; GEGENBAUR, 1878; HELLMUTH, 1902; PARKER, 1931 e STORER et al., 2000). Em outros répteis, como lagartos e cobras, eles são assimétricos (CIESLAK, 1945; BERGMAN, 1951; BRAGDON, 1953; DAS, 1960 e BETZ, 1963).

Outro elemento integrante dos órgãos genitais femininos dos répteis são os ovidutos, cuja constituição, segundo ASHLEY (1969) é de tubos largos de cor branca, dispostos ao longo do lado posterior dos ovários, podendo ser contorcidos ou retilíneos (BAROUDI, 1970), sendo que o lado direito tende a ser maior que o esquerdo, enquanto nas aves o oviduto direito é vestigial e o esquerdo é bem desenvolvido (HILDEBRAND, 1995 e DYCE et al., 1997).

PALMER e GUILLETE (1988) e GIST e CONGDON (1998) descrevem o oviduto das tartarugas formado por cinco partes: Infundíbulo, magno, istmo, útero e vagina. O mesmo acontece com a salamandra, segundo GREVEN (1998), assim como nas aves, conforme GUPTA e MAITI (1987), HILDEBRAND (1995) e DYCE et al. (1997), enquanto nas serpentes, segundo SEVER et al. (2000), os ovidutos estão divididos em quatro partes: infundíbulo anterior e posterior, útero e vagina.

Necessitando conhecer os aspectos da morfologia e do comportamento do muçuã, pesquisadores do curso de Medicina Veterinária da Universidade Estadual do Maranhão - UEMA desenvolvem pesquisas que visam contribuir para a criação e preservação destes animais, pois trata-se de uma espécie utilizada pela população da Baixada Maranhense como fonte de proteína e renda.

Nesse contexto, foram escolhidos como elementos deste estudo os órgãos genitais femininos do muçuã, com o objetivo de caracterizar a anatomia dos seus componentes, delinear sua topografia, examinando possíveis variações existentes e comparando-os com os de espécies mais próximas para, assim, contribuir com o estudo da reprodução e preservação em cativeiro desta espécie.

\section{Materiais e Métodos}

A pesquisa foi realizada no Laboratório de Anatomia Veterinária do Curso de Medicina Veterinária da Universidade Estadual do Maranhão - UEMA. Foram estudadas 10 fêmeas adultas, adquiridas mediante autorização do Instituto Brasileiro do Meio Ambiente e dos Recursos Renováveis (IBAMA), conforme processo no 020.12.002400/99-31 e licença no 002/01. Esses animais foram apreendidos em feiras livres, cabendo ressaltar que, na maioria das vezes, encontravam-se mutilados, o que impossibilitava sua reintrodução ao seu habitat natural.

Os animais foram submetidos à eutanásia com aplicação de tiopental sódico a $2,5 \%$ na dose letal de $3 \mathrm{ml}$ por via intramuscular. Após o sacrifício, caracterizado por relaxamento muscular, procedia-se à desarticulação da ponte óssea que separa o plastrão da carapaça, com o auxílio de uma serra de aço comum, removendo-se, em seguida, com um bisturi, os tecidos e a musculatura que fecham a cavidade celomática.

Após observação a fresco da topografia dos órgãos genitais femininos, as peças foram colocadas em solução aquosa de formaldeído a $10 \%$, por um período mínimo de 24 horas, para sua fixação e, com o auxílio de lupa, posterior dissecação macroscópica.

\section{Resultados}

Os resultados apresentados a partir de 10 exemplares de muçuã fêmea, adultas, com média de peso de $332,9 \mathrm{~g}$ e morfologia da carapaça de $14 \mathrm{~cm}$ por $9,2 \mathrm{~cm}$ e plastrão de $12,8 \mathrm{~cm}$ por $6,3 \mathrm{~cm}$, correspondentes respectivamente ao comprimento e largura, mostraram os órgãos genitais femininos do muçuã localizados dorsalmente no interior da cavidade celomática, constituídos por um par de ovários e um par de ovidutos, igualmente desenvolvidos, que confluem para a cloaca (FIGURA 1B).

As gônadas estão localizadas na parte caudal da cavidade celomática, sendo duas massas de coloração amarelo-ouro, compostas de vários folículos em diferentes estágios de desenvolvimento (FIGURA 1C). Estão dispostas simetricamente e fixadas ao teto da cavidade e aos rins por uma membrana de tecido conjuntivo, o mesovário (FIGURA 1D).

No que se refere à sua topografia, as gônadas femininas estabelecem relações medialmente com 0 cólon e reto, lateralmente com os ovidutos, dorsalmente com os rins, veia cava caudal, aorta caudal e teto da cavidade celomática; ventralmente, com a vesícula urinária, cranialmente com os pulmões, intestino delgado, fígado e estômago, e caudalmente com a vagina.

Os ovidutos são dois longos tubos, igualmente desenvolvidos, bastante contorcidos, que se estendem das proximidades dos ovários até se inserirem na parede lateral da cloaca.

Estão fixados por uma membrana de tecido conjuntivo, o mesoviduto, que segue ao longo da margem lateral do oviduto em direção à parede da cavidade celomática, conferindo-Ihes sustentação. Cada oviduto está dividido em cinco segmentos: infundíbulo, magnum, istmo, útero e vagina (FIGURA 1E). 
Morfologia dos órgãos genitais femininos do muçuã (Kinosternon scorpioides)

O infundíbulo é o primeiro segmento do oviduto e apresenta, na sua parte inicial, o óstio abdominal, com presença de fimbrias. O magnum, segunda parte do oviduto, forma a região produtora de albúmen, sendo seguido do istmo, parte mais estreita do oviduto.

As duas últimas partes do oviduto são o útero, que é uma parte de parede espessa e coloração escura, dotada de glândulas responsáveis pela produção da casca, e a vagina, parte final do oviduto, que mantém íntima relação com a cloaca. Nesta, há um esfíncter que tem as funções de reter os ovos até a postura, além de manter fechado o oviduto, evitando o refluxo de material da cloaca para o interior do mesmo, havendo ainda, na sua parede, uma grande concentração de glândulas produtoras de muco, responsável pela lubrificação da vagina durante a oviposição.
No segmento final do oviduto, encontra-se uma prega de tecido conjuntivo ligando as duas vaginas, e que se estende até a região de útero, denominada ligamento intervaginal.

Os ovidutos mantêm relações topográficas dorsolateralmente com a parede da cavidade pleuroperitoneal, ventralmente com a vesícula urinária e pelve, medialmente com os ovários, cranialmente com o fígado, vesícula biliar, intestino, estômago e pulmão e, caudalmente, com a cloaca .

No muçuã, assim como em outros répteis e nas aves, a cloaca é a porção terminal comum aos órgãos genitais, digestórios e urinários. Este órgão apresenta, nas suas paredes laterais, as aberturas dos ovidutos, dorsalmente o cólon, ventralmente a uretra e vetrolateralmente os ureteres (FIGURA 1F).

FIGURA 1 - FOTOGRAFIAS REPRESENTATIVAS DOS ÓRGÃOS GEITAIS DO MUÇUÃ (Kinosternon scorpioides) FÊMEA. OBSERVA-SE UM EXEMPLAR DE MUÇUÃ FÊMEA (A) NO SEU HABITAT NATURAL; EM B, NOTAM-SE OS OVÁRIOS (1), OVIDUTOS (2) E CLOACA (3); EM C, VERIFICAM-SE OS OVÁRIOS, COM VÁRIOS FOLÍCULOS (1), E SUA RELAÇÃO COM OUTROS ÓRGÃOS DA CAVIDADE CELOMÁTICA, SENDO ELES INTESTINO (2) E FÍGADO (3); EM D, EVIDENCIAM-SE O MESOVÁRIO (1) E A RELAÇÃO DOS OVÁRIOS COM OS RINS (2); EM E, OBSERVAM-SE AS DIVISÕES DO OVIDUTO DIREITO DO MUÇUÃ EM INFUNDÍBULO (1), MAGNUM (2), ISTMO (3), ÚTERO (4) E VAGINA (5); E, EM F, É POSSÍVEL NOTAR A CLOACA RECEBENDO A ABERTURA DA URETRA (1), URETERES (2), OVIDUTOS (3) E RETO (4).
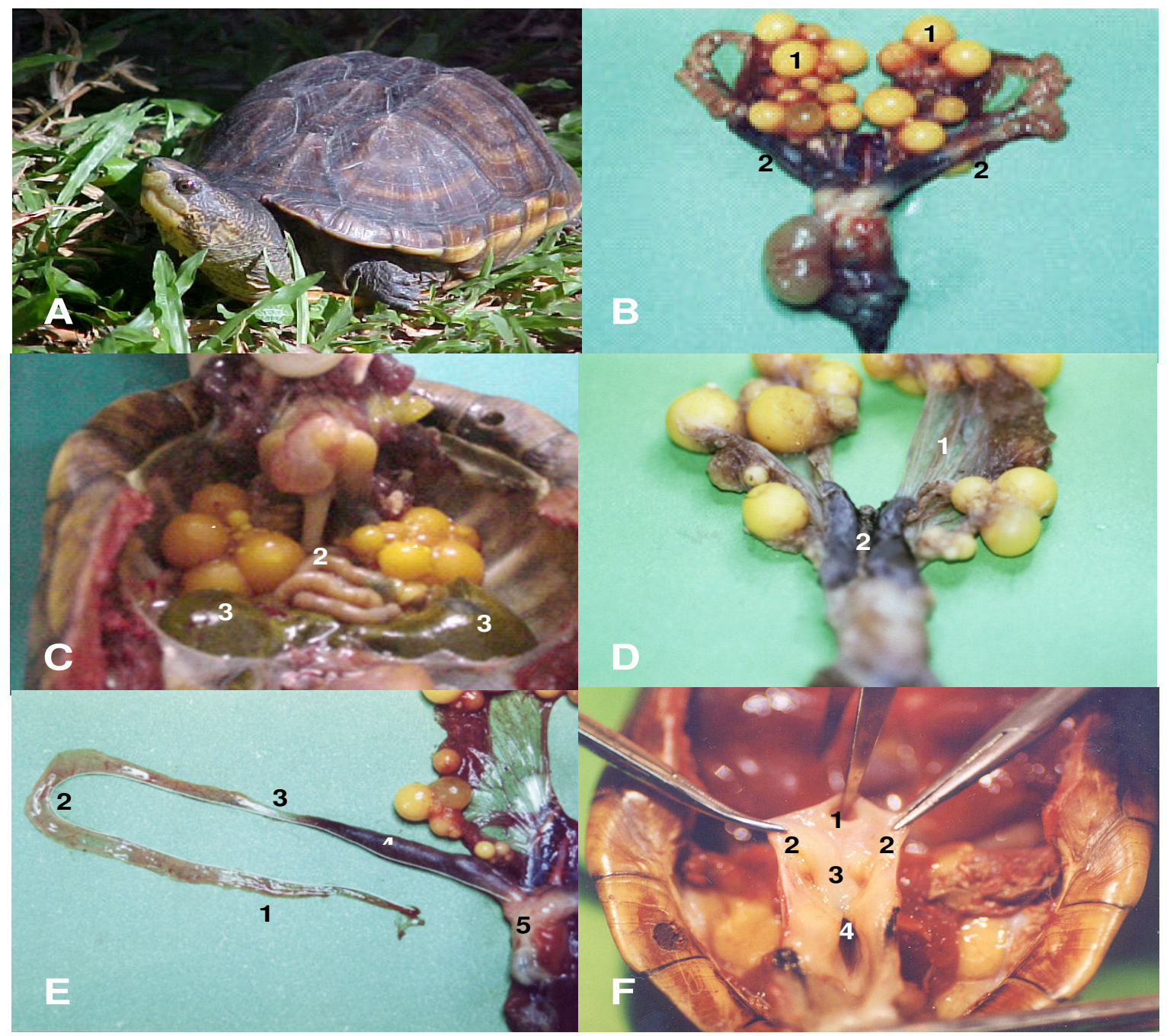


\section{Discussão}

Os órgãos genitais femininos do muçuã apresentamse constituídos por dois ovários e dois ovidutos, à semelhança dos animais répteis, segundo BAROUDI (1970), SANDLEIR (1973) e STORER et al. (2000), e das tartarugas, conforme NOBLE e NOBLE (1940). ELLENPORT (1986) e DYCE et al. (1997) relatam que apenas o lado esquerdo do aparelho reprodutor feminino das aves é desenvolvido, diferindo do que foi encontrado no muçuã, o qual apresenta estes órgãos desenvolvidos e funcionais bilateralmente.

Em todos os 10 exemplares (100\%) de fêmeas adultas de Kinosternon scorpioides, os ovários apresentaram-se sempre constituídos de massas amarelas, compostas de diversos folículos em diferentes fases de desenvolvimento, contendo uma densa quantidade de vitelo, cuja função, segundo a literatura, é servir de matéria nutritiva ao embrião ao longo do seu desenvolvimento dentro do ovo. Essa característica observada no muçuã foi também citada para os répteis por ANDREW e HICKMAN (1974), para as tartarugas por ASHLEY (1969) e para as aves por DYCE et al. (1997), sendo que todos esses animais apresentam desenvolvimento embrionário semelhante ao muçuã, ocorrendo dentro de um ovo fora do corpo da fêmea.

No que se refere à sua fixação, os ovários do muçuã estão ligados ao teto da cavidade celomática por uma membrana de tecido conjuntivo denominada mesovário. Esta fixação também foi citada por ELLENPORT (1986) e DYCE et al. (1997) para as aves, por NOBLE e NOBLE (1940) e ASHLEY (1969) para as tartarugas e BLACKBURN (1998) para os répteis esquamatos.

Quanto à posição dos ovários do muçuã na cavidade celomática, os mesmos apresentam-se dispostos simetricamente um em relação ao outro, tal como foi também descrito para as tartarugas por OWEN (1866), GEGENBAUR (1878), HELLMUTH (1902), PARKER (1931) e STORER et al. (2000). Porém, outros répteis, como lagartos e cobras, apresentam os ovários posicionados de forma assimétrica, com o direito mais cranial que o esquerdo, segundo informações de CIESLAK (1945), BERGMAN (1951), BRAGDON (1953), DAS (1960) e BETZ (1963).

BAROUDI (1970) comentou que os ovidutos dos répteis variam quanto à disposição, podendo ser retilíneo ou contorcido. Nesse aspecto, os ovidutos do muçuã apresentam-se contorcidos, com numerosas dobras, não tendo sido encontrados no formato retilíneo. Quanto ao desenvolvimento desse órgão, HILDEBRAND (1995) descreveu que, nos répteis, o oviduto direito tende a ser maior, enquanto o mesmo lado é vestigial nas aves. Ambas descrições são diferentes da que foi verificada no muçuã, que apresenta dois ovidutos de tamanho semelhante e igualmente funcionais, estando conectados à cloaca por meio da vagina.

ELLENPORT (1986) e DYCE et al. (1997) citam que a fixação do oviduto esquerdo das aves é efetuada por uma prega de tecido conjuntivo, o mesoviduto. No muçuã, este ligamento apresenta-se bilateralmente, fixando os ovidutos à parede lateral da cavidade celomática. Existe ainda no muçuã, entre as duas vaginas, uma prega de tecido conjuntivo, o ligamento intervaginal, cuja finalidade é uni-las, evitando uma distensão excessiva quando os úteros estão repletos de ovos. Não foi observado na literatura nenhuma alusão a esta prega em outros répteis; porém, por analogia, pode-se admitir que se tratam de membranas homólogas aos ligamentos intercornuais da vaca, citados por DYCE et al. (1997).

Os ovidutos do muçuã encontram-se divididos em cinco segmentos, infundíbulo, magnum, istmo, útero e vagina, à semelhança do oviduto esquerdo das aves, citado por GUPTA e MAITI (1987) e DYCE et al. (1997), e das tartarugas, referidas por GIST e CONGDON (1998) e PALMER e GUILLETE (1988). Nas serpentes, SEVER et al. (2000) descrevem o oviduto como formado por quatro partes, designadas infundíbulo anterior, infundíbulo posterior, útero e vagina.

Nas salamandras, segundo GREVEN (1998), assim como no muçuã, o infundíbulo apresenta um óstio abdominal, com presença de fímbrias, as quais favorecem a captação dos ovócitos liberados pelos ovários, direcionando-os para o interior do oviduto.

O magnum, segundo segmento do oviduto do muçuã, forma a região produtora de albúmen, tal como foi descrito por GIST e CONGDON (1998) para as tartarugas. Enquanto o útero possui glândulas responsáveis pela confecção da casca do ovo, sendo a mesma função citada por PALMER e GUILLETE (1988) para a tartaruga Gopherus polyphemus.

$\mathrm{Na}$ porção final do oviduto do Kinosternon scorpioides, especificamente a região da vagina, existe um esfíncter que tem como função reter os ovos. Nesta região observa-se, ainda, a presença de glândulas que servem para lubrificar a vagina durante a oviposição, além de produzir um tampão que, juntamente com o esfíncter vaginal, mantém os ovidutos fechados, evitando assim o refluxo oriundo da cloaca, com a conseqüente contaminação por resíduos metabólitos de órgãos urinários e digestivos. Estes achados se assemelham aos descritos por PALMER e GUILLETE (1988) para a tartaruga Gopherus polyphemus.

Os ovidutos do muçuã, especificamente as duas vaginas, desembocam lateralmente na cloaca, à semelhança do que é observado nas aves para o oviduto esquerdo, conforme citado por DYCE et al. (1997).

Com base nos achados obtidos segundo a metodologia proposta, conclui-se que os órgãos genitais femininos do muçuã (Kinosternon scorpioides) são funcionais bilateralmente, sendo constituídos por um par de ovários e um par de ovidutos, os quais encontramse divididos em cinco segmentos: infundíbulo, magno, istmo, útero e vagina, esta última confluindo para a cloaca. 
Morfologia dos órgãos genitais femininos do muçuã (Kinosternon scorpioides)

\section{Referências}

ANDREW, W; HICKMAN, C.P. Histology of the vertebrates. Saint Louis: The C. V. Mosby Company, 1974. p.225.

ASHLEY, L.M. Laboratory anatomy of the turtle. 6th ed. Dubuque: W.C. Browun Company Publishers. 1969. 47 p.

BAROUDI, R. Elementos da zoologia. 6. ed. São Paulo: Nobel, 1970. p.125-152.

BERGMAN, R. A. M. The anatomy of Natrix vittata. Zoology. v.31. p.13-24, 1951.

BETZ, T. W. The Gross ovarian morphology of the diamond-backed water snaker Natrix rhombifera, during the reproductive cycle. Copeia. v.4, p.692-697. 1963.

BLACKBURN, D.G. Structure, function and evolution of the oviducts of squamate reptiles, with special reference to viviparity and placentation. The Journal of Experimental Zoology. v.282, p.560-617, 1998.

BRAGDON, D.E. A contribuition to the surgical anatomy of the water snaher Natrix sipedon sipedon; the location of the visceral endocrine organs with reference to ventral scutellation. Anatomical Record, v.117, p.145-161,1953.

CIESLAK, E. S. Relation between the reproductive cycle and the pituitary gland inthe snake Thamnophis radix. Physiology and Zoology. v.18, p.299-329, 1945.

DAS, S.M. A comparative functional study of the urinogenital system in Uromastyx hardwickii Gray (sand lizard), Ptyas mucosus (rat snaker) and Eryx conicus (sand boa). Proceedings National. Academy Sciences. v.30, p.5978, 1960.

DYCE, K.M.; SACK, W.O.; WENSING, C.I.G. Tratado de anatomia veterinária. Rio de Janeiro: Guanabara Koogan, 1997. p. 645-647.

ELLENPORT, C.R. Aparelho urogenital geral. In: GETTY, R. Anatomia dos animais domésticos. 5.ed. Rio de Janeiro: Guanabara Koogan, 1986. p.1813-1828.

GEGENBAUR, C. Elements of comparative anatomy. Translated by F. J. Bell and E. Ray Lankester. London: Macmilian. 1878. s.p.

GIST, D.H.; CONGDON, J.D. Oviductal sperm storage as a reproductive tactic of turtles. The Journal of Experimental Zoology. v. 282, p.526-534, 1998.
GREVEN, H. Survey of the oviduct of salamandrids with special reference to the viviparous species. The Journal of Experimental Zoology. v.282, p.507-525, 1998.

GUPTA, S. K.; MAITI, B. R. Seasonal changes in the oviduct of the pied myna (Aves: Sturnidae). Journal of Morphology. v.194, n. 3, p.247-263. 1987.

HELLMUTH, H. Die Schildkröten und krokodile. Journal of Morphology. v.30, p.582-613, 1902.

HILDEBRAND, M. Análise da estrutura dos vertebrados. São Paulo: Atheneu, 1995. p.323-328.

NOBLE, G.A.; NOBLE, E.R. A brief anatomy of turtle. Stanford University Press, Stanford University California. London: Hamphrey Oxford University Press, 1940. 49p.

ORR, R. T. Biologia dos vertebrados. 5 ed. São Paulo: Roca, 1986. p.95-128.

OWEN, R. On the anatomy of vetebrate. London: Longmans, Green, \& Co. 1866. s.p.

PALMER, B. D.; GUILLETE, L. T. Jr. Histology and fuctinonal morphology of the female reproductive tract of the tortoise Gopherus polyphemus. America Journal of Anatomy. v.183, n.3, p.200-211. 1988.

PARKER, G.H. The passage of sperms and eggs through the oviducts in terrestrial veterbrates. Biological Sciences. v.219. p.381-419. 1931.

PEREIRA, J.G. Estudo histológico e histoquímico do tubo digestivo e do pâncreas do Kinosternon scorpioides Linnaeus, 1756 (Reptilia, Chelonia, Kinosternidae), muçuã. Viçosa, 2000. 148p. Dissertação (Mestrado em Medicina Veterinária) - Universidade Federal de Viçosa.

SANDLEIR, R.M.F.S. The reproduction of vertebrates. New York: Academic press, 1973. p. 56-59.

SEVER, D.M.; RYAN, T.J.; MORRIS, T.; PATTON, D.; SWAFFORD, S. Ultrastructure of the reproductive system of the black swamp snake (Seminatrix pygaea). II. Annual oviductal cycle. Journal of Morphology. v.245, n.2, p.146160. 2000.

STORER, T.I.; USINGER, R.L.; STEBBINS, R.C.; NYBAKKEN, J.W. Zoologia geral. 6 ed. v. 8. São Paulo: Companhia Editora Nacional, 2000. p. 642-654.

Recebido para publicação: 27/02/2006 Aprovado: $30 / 08 / 2006$ 